\title{
Security Analysis of a Multi-factor Authenticated Key Exchange Protocol
}

\author{
Feng Hao and Dylan Clarke \\ School of Computing Science \\ Newcastle University \\ \{feng.hao,dylan.clarke\}@ncl.ac.uk
}

\begin{abstract}
This paper shows several security weaknesses of a MultiFactor Authenticated Key Exchange (MK-AKE) protocol, proposed by Pointcheval and Zimmer at ACNS'08. The Pointcheval-Zimmer scheme was designed to combine three authentication factors in one system, including a password, a secure token (that stores a private key) and biometrics. In a formal model, Pointcheval and Zimmer formally proved that an attacker had to break all three factors to win. However, the formal model only considers the threat that an attacker may impersonate the client; it however does not discuss what will happen if the attacker impersonates the server. We fill the gap by analyzing the case of the server impersonation, which is a realistic threat in practice. We assume that an attacker has already compromised the password, and we then present two further attacks: in the first attack, an attacker is able to steal a fresh biometric sample from the victim without being noticed; in the second attack, he can discover the victim's private key based on the Chinese Remainder theorem. Both attacks have been experimentally verified. In summary, an attacker actually only needs to compromise a single password factor in order to break the entire system. We also discuss the deficiencies in the Pointcheval-Zimmer formal model and countermeasures to our attacks.
\end{abstract}

\section{Introduction}

Authenticated Key Exchange (AKE) is a fundamental security protocol for almost all secure communication systems. Depending on how the "authentication" is defined, AKE schemes are generally divided into two categories: Passwordbased Authenticated Key Exchange (PAKE) and PKI-based Authenticated Key Exchange [7. In the former case, the authentication is based on the knowledge of a shared password, without requiring any Public Key Infrastructure (PKI). In the latter case, each party possesses a unique pair of the public and private keys. The authentication is based on the possession of the private key, which is usually stored in a tamper resistant device. A PKI is needed to securely distribute authentic public keys to all users [18.

While the above two AKE categories correspond to something you know (i.e., a password) and something you have (i.e., a secure token that stores a private key) respectively, there is a third authentication factor: namely, something you are 
(i.e., biometrics). Biometrics are an advanced authentication mechanism, which works by measuring a person's unique behavioral or physical characteristics [2]. The security of biometrics largely depends on whether a trusted path exists, which ensures the biometric sample is freshly obtained from the live subject. Such a trusted path can be realized, for example by enforcing supervision in a controlled environment (e.g., airport). In an unsupervised environment, the security will have to depend on the liveness detection features embedded with the biometric scanning equipment [18]. Assuming a trusted path already in place, researchers have made progress in designing biometrics-based AKE schemes [3, 5, 6].

So far all the above-mentioned AKE schemes are based on a single factor. In recent years, Multi-Factor Authenticated Key Exchange (MF-AKE) has emerged as an active research topic 10 14, 18 23. The rationale is to improve single factor based AKE by combining two or even more factors in one system. This is a worthy goal, but extra caution should be taken. The past thirty years of research in the area of authenticated key exchange has proved that it is incredibly difficult to get even a single factor based AKE scheme right [4]. Designing a multi-factor AKE protocol can only be harder.

Many MF-AKE protocols have been proposed - and subsequently broken. For example, in 2010, Lee et al. proposed a two-factor AKE protocol that combines a smartcard and a password [11]. But a year later, their protocol was found insecure: the compromise of the smartcard factor breaks the entire scheme 21.

$\mathrm{Xu}$ et al. proposed a similar two-factor AKE protocol based on a smartcard and a password with "formal security proofs" in [23]. Within a year, their protocol was broken and a patched protocol was proposed in [19]. Yet the patched protocol was shortly found insecure [20]. Li and Hwang proposed a different type of twofactor AKE protocol, which consists of biometrics and a smart card [13. In less than a year, their scheme was broken 14. These examples show that MK-AKE is still a young field; more research is very much needed.

Recently at ACNS'08, Pointcheval and Zimmer proposed the first MultiFactor Authenticated Key Exchange protocol that combines all three factors in one system: a password, a smartcard and biometrics [18. Furthermore, the authors defined a formal model and formally proved that an adversary had to break all three factors in order to win. Unfortunately, we find their protocol vulnerable, as we explain below.

\section{Pointcheval-Zimmer Protocol}

In this section, we will describe Pointcheval-Zimmer's Multi-Factor Authenticated Key Exchange scheme. We will follow the original notations in [18] as closely as possible.

\subsection{Notation}

The client $\mathcal{C}$ owns a tuple $t_{\mathcal{C}}=\left(W_{\mathcal{C}}^{\prime}, \mathrm{sk}_{\mathcal{C}}=\mathrm{x}_{\mathcal{C}}, \operatorname{pwd}_{\mathcal{C}}\right)$ where $W_{\mathcal{C}}^{\prime}$ is a biometric, $\mathrm{sk}_{\mathcal{C}}$ a private key and $\mathrm{pwd}_{\mathcal{C}}$ a password. The iris code is used in [18] as a specific example for biometrics. 
The server $\mathcal{S}$ holds a list of tuples for each client $t_{\mathcal{S}}=<t_{\mathcal{S}}[\mathcal{C}]>$, where $t_{\mathcal{S}}[\mathcal{C}]$ is a transformed-tuple of $t_{\mathcal{C}}$. More specifically, $t_{\mathcal{S}}[\mathcal{C}]$ contains the following information about the $\operatorname{client} \mathcal{C}$ :

- The client's public key $h=g^{x_{\mathcal{C}}}$.

- An encrypted copy of the iris-code template that was enrolled during registration. The template is denoted as $W_{\mathcal{C}}=\left(W_{i}\right)_{i \leq N}$, where $W_{i}$ is the $i$-th bit of $W_{\mathcal{C}}$ and $N$ is the number of bits of an iris code. The ciphertext is obtained by using the El Gamal encryption algorithm; the result is $\left(g^{r_{i}}, h^{r_{i}} \cdot g^{W_{i}}\right)_{i}$, where $r_{i}$ is a random element in $\mathbb{Z}_{q}$.

- The client's password pwd $\mathrm{C}_{\mathcal{C}}$.

\subsection{Description of Protocol}

The protocol is based on a cyclic group with parameters $(p, g, q)$. The $p$ and $q$ are big prime numbers, and $q \mid p-1$. Let $\mathbb{G}_{q}$ be a subgroup in $\mathbb{Z}_{p}^{*}$ with prime order $q$, and $g$ be its generator. In addition, the protocol defines two random elements in $\mathbb{G}_{q}$, namely $u$ and $v$. Figure 1 specifies how the protocol works. The symbols used in the figure should be self-explanatory 1 .

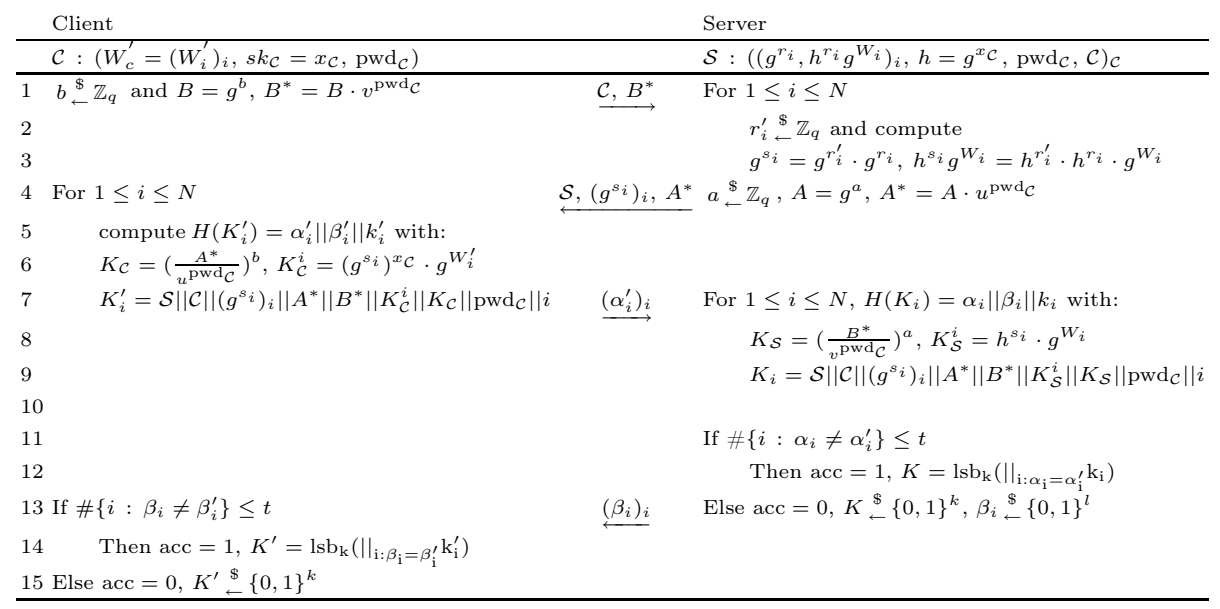

Fig. 1. Pointcheval-Zimmer protocol

In [18], the authors also suggest practical parameters for a real-world implementation. They assume an iris scan has $N=1024$ bits. A value of $t=300$ is defined as the threshold, so two iris codes with less than $t$ disparate bits

${ }^{1}$ The original specification in in $[18$ does not explicitly explain the meaning of lsb in Line 12 and 14. We assume lsb refers to "least significant bits" and we interpret it as a key derivation function that derives a session key from raw keying materials. This ambiguity does not affect our security analysis however. 
(i.e., Hamming distance) are considered belonging to the same eye; otherwise, they are regarded from different eyes. Furthermore, the authors use $l$ to denote the bit lengths of $\alpha, \alpha^{\prime}, \beta, \beta^{\prime}$ (see line 5 and 7 in Figure 1). That is: $l=\left\|\alpha^{\prime}\right\|=\left\|\beta^{\prime}\right\|=\|\alpha\|=\|\beta\|$.

The value of $l$ is critical to the correctness of the protocol. For small values of $l$, say $l=1$, then the protocol will be guaranteed to fail even between two honest players. To ensure a successful honest execution of the protocol, the value $l$ must not be small. Pointcheval and Zimmer recommend $l=24$, and they estimate that with this parameter, the probability for a successful execution between two honest players is $1-2 \cdot t \cdot 2^{-24}=1-2^{-14}$.

Pointcheval and Zimmer also define a formal model to prove the security of the protocol [18. The model assumes the adversary is able to corrupt a client $\mathcal{C}$ in the following ways: by compromising the password $\operatorname{pwd}_{\mathcal{C}}$, by stealing the private key $\mathrm{sk}_{\mathcal{C}}$, or by spoofing biometrics $W_{\mathcal{C}}$. Under this model, the authors formally prove that an attacker has to compromise all three factors in order to win. However, the formal model only considers the unilateral authentication from the client to the server. In other words, it implicitly assumes the server is honest. The authors of 18 acknowledge that "this can be seen as a strong limitation", but on the other hand, they argue "it is not in practice: if the password and the secret keys are compromised, an adversary can easily play the role of the server". We find this justification weak and unclear; in particular, the authors do not explicitly explain what will happen if the attacker is able to impersonate the server to the client (e.g., in a man-in-the-middle attack). We fill the gap by analyzing this threat in the following section.

\section{Attacks}

When the attacker is able to impersonate the server (which is a realistic threat in practice), we show the Pointcheval-Zimmer protocol is insecure. First of all, we assume that the attacker has compromised the client's password (say by phishing). Then, we show how the attacker is able to subsequently compromise the other two factors: the biometrics and private key, hence breaking the entire system.

\subsection{Stealing Biometrics}

First, we show how the compromise of the password factor will lead to the breach of the biometrics factor. Figure 2 shows how the attack works. As shown in Line 2 , the attacker selects random values for $s_{i}$ and computes $g^{s_{i}}$ accordingly. This is a clear deviation from the original specification, because if the server had honestly followed the specification in computing $g^{s_{i}}=g^{r_{i}^{\prime}} \cdot g^{r_{i}}$, it will not have knowledge of the exponent of $g^{s_{i}}$. But in this case, the server (attacker) knows the exponent (which allows carrying out the subsequent attack). This deviation is undetectable to the client.

One principle in designing robust security protocols is "never let yourself be used as an oracle by your opponent" [1]. Unfortunately, in this case, the client has 


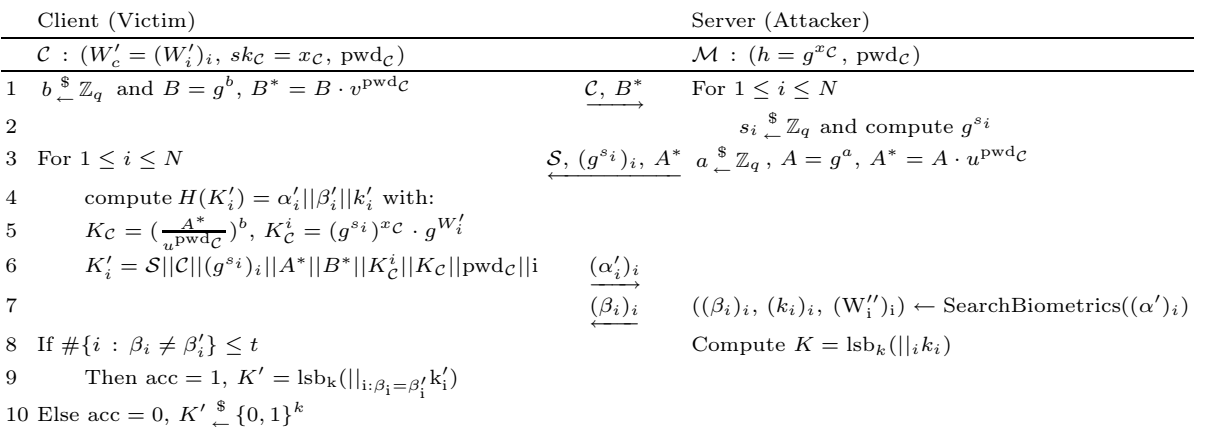

Fig. 2. Attack 1: stealing fresh biometrics from the client without being detected

made itself an oracle to the attacker. After receiving the data from the attacker, the client proceeds to compute values of $\left(\alpha_{i}^{\prime}\right)_{i}$ and sends them over to the server. Those values will allow the attacker to discover the biometric sample from the user, as described in Algorithm 1

The recovered sample from Algorithm 1 is high-quality biometric data. By "high-quality", we mean the recovered sample $\left(W_{i}^{\prime \prime}\right)_{i}$ is extremely close to the client's sample $\left(W_{i}^{\prime}\right)_{i}$, which is freshly acquired in a favorable supervised condition. The exact difference between $\left(W_{i}^{\prime \prime}\right)_{i}$ and $\left(W_{i}^{\prime}\right)_{i}$ depends on the parameter $l$ (which is the bit length of $\alpha, \alpha^{\prime}, \beta, \beta^{\prime}$ ). If we take $l=24$ as recommended in [18], the probability of each bit in $W_{i}^{\prime \prime}$ being correct (i.e., it equals the bit in $W_{i}$ ) is $p=1-2^{24}$. Hence, the probability of all $N=1024$ bits in $W_{i}^{\prime \prime}$ being correct is $p^{N}=99.994 \%$. With an almost identical biometric sample, it is trivial for the attacker to compute $\left(\beta_{i}\right)_{i}$ so that he can successfully finish the rest of the protocol (see Algorithm 1). In reality, having two identical biometric samples normally suggests a replay attack, so the attacker may randomly corrupt some (up to $t$ ) of the $\beta_{i}$ values to artificially make the biometric matching look "fuzzy". The same attack also applies to the three-party extension of Pointcheval-Zimmer's protocol, which was proposed in [15].

\subsection{Disclosing Private Key}

In the second attack, we show how the attacker is able to recover the client's private key, based on a compromised password and stolen biometrics (obtained from the first attack).

The attack is possible because the client is not required to perform public key validation on the data received from the server (Line 4-7 in Figure 1). Many protocols omit the step of public key validation in order to increase the protocol efficiency. But this is often done at the expense of security. One wellknown example is HMQV, which "provably" drops public key validation based on

${ }^{2}$ In the three-party extension of the Pointcheval-Zimmer protocol [15, there is no public key validation either. So the same attack applies. 


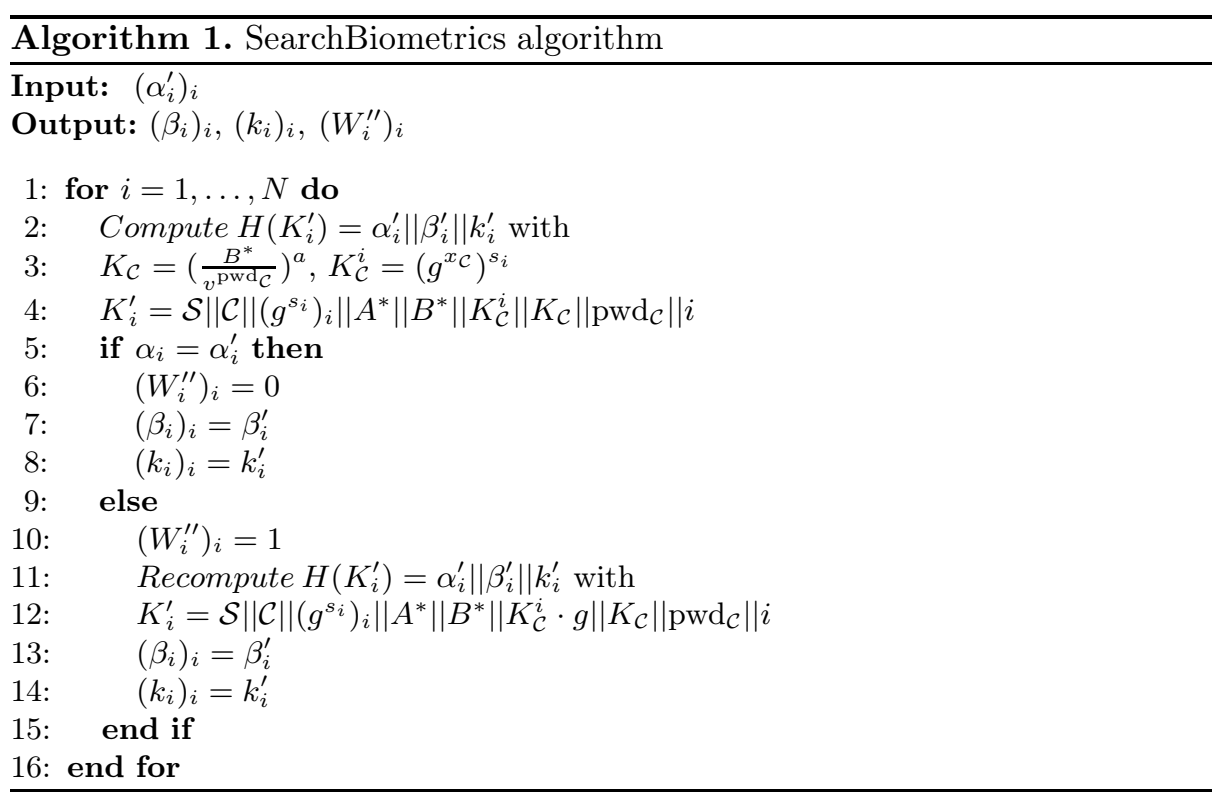

a formal model and security proofs [9]. However, Menenzes et al. subsequently pointed out the flaws in the formal model and attacks on HMQV [17]. Their paper highlights the importance of performing the public key validation. In [18], Pointcheval and Zimmer defined a formal model and provided formal security proofs for their MK-AKE protocol. However, the formal model in [18] implicitly assumes the server is honest, which is not a valid assumption.

The attack works as follows. Instead of computing $g^{s_{i}}$ as in the original protocol (Line 3 in Figure 1), the attacker selects small subgroup elements in $\mathbb{Z}_{p}^{*}$ and sends them to the client. We use $\mathbb{G}_{s}$ to denote a small subgroup of prime order $s$. Let us take $i=1$ as an example. Let $b_{1}$ be a generator of $\mathbb{G}_{s}$ (i.e., an arbitrary non-identity element). After receiving $b_{1}$, the client proceeds to compute $\alpha_{1}^{\prime}$ as specified in the original protocol and sends it to the server. We know $W_{1}=W_{1}^{\prime}$ with a high probability (say 90\%). For simplicity of illustration, we first assume $W_{1}=W_{1}^{\prime}$. The attacker knows all the concatenated items in $K_{i}^{\prime}$ (Line 7 in Figure 1), except $K_{\mathcal{C}}^{i}$. Based on $W_{1}=W_{1}^{\prime}$ and the fact that $b_{1}^{x_{\mathcal{C}}} \bmod p$ falls within a small range, the attacker can easily obtain the value of $a_{1}=b_{1}^{x_{\mathcal{C}}} \bmod p$ by exhaustive search (i.e., against the value of $\alpha^{\prime}$ ). In the subsequent step, the attacker can compute $x_{\mathcal{C}}$ mods - once again by exhaustive search (based on $a_{1}, b_{1}$ and $p$ ). Since $b_{1}$ is a generator in $\mathbb{G}_{s}, b_{1}^{x_{\mathcal{C}}}$ also falls within the same small subgroup. Through exhaustive search, the attacker can obtain $x_{\mathcal{C}}$ mod s. By repeating the same procedure for different subgroups, the attacker can recover more secret bits of the private key. Depending on the group setting, it is possible to recover a full copy of the private key based on the Chinese Remainder theorem (e.g., see a concrete implementation of the attack in Appendix A). 
In the above analysis, we have assumed $W_{1}=W_{1}^{\prime}$, but in reality the equality only holds for a probability say $90 \%$. This problem can be easily addressed by exploiting the large amount of redundancies in an iris code. Instead of sending one $b_{i}$ value, the attacker could send several $b_{i}$ values from the same small subgroup $\mathbb{G}_{s}$. For example, with $30 b_{i}$ values, there will be on average $30 \times 0.9=$ 27 results that give the same $x_{\mathcal{C}} \bmod$ s. This removes any uncertainty due to the fuzzy nature of biometrics.

\subsection{Combining Two Attacks}

It is easy to combine the two attacks together. For that, we need to modify the first attack slightly: after successfully stealing a biometric sample, the adversary sends to the client random $\beta_{i}^{\prime}$ values to trigger a "rejection". Because the $\beta_{i}^{\prime}$ values are random, the matching at the client will fail. However, the failure will hardly raise any suspicion from the client, as any biometric system has a non-zero false rejection rate in the real-world operation 3. Most likely, the user will be prompted to try again (and be ready to be more cooperative with the iris photo-taking). In the second attempt, the attacker will send small subgroup elements to discover the user's private key. Subsequently, the attacker will send correct $\beta^{\prime}$ values to trick the client into believing that the second attempt is successful.

Thus, with a stolen password, the attacker has successfully compromised the user's two other factors, hence breaking the entire system.

\section{Discussion}

Between the two attacks, the second attack might look more damaging, but it is easier to fix. We can address it by adding public key validation in the protocol. However, this will significantly decrease the computational efficiency of the original protocol, as it normally takes a full exponentiation to verify if the received public key is a valid element in the correct subgroup of $\mathbb{Z}_{p}^{*}$. Nonetheless, our attack shows that this step is necessary (more explanation about the importance of public key validation can be found in [17]).

The first attack indicates a more fundamental flaw with the protocol. The question concerns the exact role of biometric authentication in the PointchevalZimmer protocol. Assume the attacker has compromised the password and the private key, but he does not have user's biometric data. What kind of security assurance can the remaining biometrics factor provide? Almost none - based on the following observations. First, the client cannot safeguard the biometrics. As shown in Section 3.1 the protocol allows a remote attacker to steal the user's freshly obtained biometric sample without the user's awareness at all. Second, the encryption of biometrics at the server does not really preserve the privacy of biometrics as claimed in [18. The attacker will target the weaker part in

${ }^{3}$ Even with two perfectly matching biometric samples, a false reject may still occur in the Pointcheval-Zimmer scheme (due to the deficiency in the engineering design of the protocol) with a probability $2^{-14}$ for $l=20$ (see [18]). 
the system - the client - to steal a biometric sample in the plaintext. Third, although by design biometric matching is done at the server, the result of the matching cannot be securely sent back to the client. As demonstrated in Section 3.1 the attacker is able to arbitrarily manipulate the matching outcome. All these suggest a fundamental problem with the protocol, which seems not easily fixable with the current structural design of the protocol. More research on this is much needed (e.g., some techniques in biometrics-based AKE [3,5, 6] may be applicable to address this issue).

The above flaw was missed by the formal analysis in 18, because the authors assume biometrics as "fully public". Their justification is that the "the opposite assumption is not reasonable in practice", which refers to the treatment of biometrics as "fully secret" in 3,5, 6. However, "biometric applications lie between the extremes of secret data and fully public data" 88. Indeed, it is possible to steal users' fingerprints that were left on the keyboard, or surreptitiously photograph their irises using hidden cameras. But, samples stolen this way tend to be of poor-quality [2]. Getting high-quality biometric samples usually requires user cooperation and a favorable environment: e.g., proper posture, distance to the camera and lighting etc. Stealing such samples without being detected by the user is not easy. When designing biometrics-based protocols, it is prudent not to rely on the secrecy of biometrics, but on the other hand, it is obviously a security flaw to give away freshly acquired biometric samples to a remote attacker.

\section{Conclusion}

In this paper, we described two attacks on Pointcheval-Zimmer's Multi-Factor Authenticated Key Exchange (MF-AKE) protocol. In the first attack, we showed how an attacker could make use of a stolen password to subsequently compromise the biometrics factor without being detected. This violates the privacy of biometric data. In the second attack, we showed how an attacker could further discover the user's private key by exploiting the small subgroup confinements. This attack breaches the presumed tamper-resistance of a secure token that stores the private key. In summary, the attacker only needs to compromise a single password factor in order to compromise the three-factor AKE protocol. These attacks are rooted in the deficiency of Pointcheval-Zimmer's formal security model, and highlight the importance of making valid assumptions in the model.

Acknowledgement. We sincerely thank Pointcheval and Zimmer for quickly and frankly acknowledging our attacks and the deficiencies in their formal model, and also for confirming our suggested countermeasures.

\section{Appendix A: An Implementation of Small Subgroup Confinement Attack on Pointcheval-Zimmer's Protocol}

We provide implementation details about the second attack below. (We have also experimentally verified the first attack, but since that implementation is straightforward, we do not include the details in the paper.) 


\section{Group Parameters}

Let us assume a cyclic group $\mathbb{Z}_{p}^{*}$ that has a subgroup of prime order $q$. Hence, $q \mid p-1$. Let $g$ be a generator, $g^{q} \bmod p=1$. As a specific example, we define a 512-bit $p, 160$-bit $q, 512$-bit $g$ with the following values (in the hex format).

$$
\begin{aligned}
p= & f 95 b 8 b 2 f 45 b 3016 e f b 6 e c 51 d 342931 a e a 4 a 5 f 4516 d 15 c 4 e d 2 c f 79 e 4 d 318 \ldots \\
& e 28837989 b e d c b e 4 c e 8693 f 68 d e 6 b 72 b 1 f 74 c 8 e 109 b c 9155 f 5 d 2 d 65 e 9 f 6 d \ldots \\
& 091 e 7 f 79 b \\
q= & e 80 f 99 e 4981 e e 1 e a c 37 d 8 f 0 b f 707 b 2067 f 6 f e 8 c f \\
g= & 33 c 65 b 25 a d 4 c 47 a c 067083 b 7 f 2 a c f 53 e d 3 a 053 d b e 508 a c b a b e 179029 d a d \ldots \\
& 77 a 04 c 0953 c 1 d b c e 02 c e 2 f 8 c f 5 b 030 a 36 d e 7868 b 7434194816 d b e 7 d a 920 \ldots \\
& 13 b c 4696 d
\end{aligned}
$$

Note that this (artificial) example is for illustration only. In practice, the bit lengths of $p$ and $q$ are normally much longer. In addition, we assume $\mathbb{Z}_{p}^{*}$ has several small subgroups of primer order $s_{i}$, so $s_{i} \mid p-1$. The hex values of $s_{i}$ are given in Table 1. Except $s_{1}$, all other $s_{i}$ are 21-bit long.

Table 1. Orders of small subgroups of $\mathbb{Z}_{p}^{*}$

\begin{tabular}{|c|c|c|c|c|c|c|c|c|c|}
\hline$i$ & 1 & 2 & 3 & 4 & 5 & 6 & 7 & 8 & 9 \\
\hline$s_{i}$ & 2 & $15 a 661$ & $1182 b b$ & $12 b 357$ & $1 f a 9 e 7$ & $1 f 1 c 9 f$ & $1 c 58 b 7$ & $16 b 6 b 3$ & $1727 c 3$ \\
\hline
\end{tabular}

\section{Small Subgroup Confinement Attack}

To demonstrate how an adversary can recover the private key, we first define a random 160-bit private key in the range of $[0, q-1]$ :

$$
x=538 b 2 f 452 c 20 f 9 c d 7 e 356455 e 2 a e 66 e 9924 d d d 5 d
$$

By exploiting the small subgroup confinement, the adversary can obtain $a_{i}=$ $b_{i}^{x} \bmod p$ for each of the small subgroups with prime orders $s_{i}$. Furthermore, he can obtain $c_{i}=x \bmod s_{i}$ through exhaustive search. Table 2 shows the results of $a_{i}$ and $c_{i}$ for each of the small subgroups $\mathbb{G}_{s_{i}}$. On average, it takes about 68.5 seconds to find $c_{i}$ using exhaustive search, on a $2.93 \mathrm{GHz}$ desktop PC with $4 \mathrm{~GB}$ memory.

With the $c_{i}$ values, the private key can be recovered based on the Chinese Remainder theorem [16. For example, we could apply Gauss's algorithm to solve the simultaneous congruences problem as follows. Let $n=s_{1} \cdot s_{2} \cdot s_{3} \cdot s_{4} \cdot s_{5} \cdot s_{6}$. $s_{7} \cdot s_{8} \cdot s_{9}$. Then $x=\sum_{i} c_{i} N_{i} M_{i} \bmod n$ where $N_{i}=n / s_{i}$ and $M_{i}=N_{i}^{-1} \bmod s_{i}$. In this specific example, $n$ (169-bit) $>q(160$-bit), so we are able to recover the full private key. It takes merely 1 millisecond to obtain the following result using Gauss's algorithm.

$$
\begin{aligned}
x^{\prime} & =538 b 2 f 452 c 20 f 9 c d 7 e 356455 e 2 a e 66 e 9924 d d d 5 d \bmod n \\
& =538 b 2 f 452 c 20 f 9 c d 7 e 356455 e 2 a e 66 e 9924 d d d 5 d \bmod q
\end{aligned}
$$


Table 2. Exhaustive search among the small subgroup confinements

\begin{tabular}{|c|c|c|c|}
\hline$i$ & $a_{i}=b_{i}^{x} \bmod p$ & $c_{i}=x \bmod s_{i}$ & $\begin{array}{l}\text { Time for exh } \\
\text { search }(\mathrm{ms})\end{array}$ \\
\hline \multirow{2}{*}{1} & f95b8b2f45b3016efb6ec51d342931aea4a5f4516d15c4ed2cf79e4d318e2883 & \multirow{2}{*}{1} & \multirow{2}{*}{0} \\
\hline & 7989bedcbe4ce8693f68de6b72b1f74c8e109bc9155f5d2d65e9f6d091e7f79a & & \\
\hline \multirow{2}{*}{2} & 791778359b242e573617fff6735703be986dd9a6271be8b413381f4211ffbc0cb & \multirow{2}{*}{122156} & \multirow{2}{*}{124854} \\
\hline & 6cb2da17c880a115e223752cb431708c4a64d68cbd8109c7a2e31e434839682 & & \\
\hline \multirow{2}{*}{3} & 133023e42630ff22b9f9fb3e6a1ceeddf2d7fc1014fcab33eeedc1af416951c2c9 & \multirow{2}{*}{$517 \mathrm{f} 1$} & \multirow{2}{*}{31481} \\
\hline & 099d8fef275408d1f82c7090cd72744a260685381a15cc8e58bdd052bb1928 & & \\
\hline \multirow{2}{*}{4} & 93358cdb4153463b93f0525d6819426b7b4c0fcb5905cf10db5f39eeea68a879 & \multirow{2}{*}{$42 \mathrm{cb} 2$} & \multirow{2}{*}{25271} \\
\hline & c8ad548cef72476671ff2805e1cbf8644f39f2d4fc71570522e4e89784a0a0aa & & \\
\hline \multirow{2}{*}{5} & f517a5a96bd4a92af483727dd0c73c251ac159056ec5b2eea90854379ba8344 & \multirow{2}{*}{$\mathrm{e} 0125$} & \multirow{2}{*}{93050} \\
\hline & cc41e641d9e84ec319fd4ab545e038cba799972a2db93b2bf315fb62b08402cb & & \\
\hline \multirow{2}{*}{6} & e1895eb12f66645c2359ad6185d85fb02f39ddd3b2e80392c3f53ffc5ebcb23a9 & \multirow{2}{*}{$15 \mathrm{~d} 34$} & \multirow{2}{*}{7544} \\
\hline & 81eb1d9cfd7a8eeea8a13e83af81a726280fcd7d545450fb6871786f9e2ebe6 & & \\
\hline \multirow{2}{*}{7} & 8dc566a29c84977ceb1e1a466321859fe3022f7ab3adae44ead9d8b7c2dc5461 & \multirow{2}{*}{121427} & \multirow{2}{*}{121793} \\
\hline & b1ea9441b19425c13da5b7c998ea7fbe41aaea70177118b37438c5f36cad85d2 & & \\
\hline \multirow{2}{*}{8} & d73e16cf041ecd7c9c73d6414ad01b0ce85deaedbcf6834591f373091a51903 & \multirow{2}{*}{ ef2c6 } & \multirow{2}{*}{99664} \\
\hline & 1cbb4aebe31fd56afab5750226584a762eaf7ffef0f5d7e2f940e27ea8d8b0d41 & & \\
\hline \multirow{2}{*}{9} & dbdb75657da4a1e4312f17b7519eff2fe2c0b0b2fbe225482e3f78f73530c545a & \multirow{2}{*}{$10 \mathrm{c} 700$} & \multirow{2}{*}{112565} \\
\hline & 3c8cd4d6fa0abd3f27058090cd1992263e72f16e47b7256f916d4a20201e5c0 & & \\
\hline
\end{tabular}

\section{References}

1. Anderson, R.J., Needham, R.: Robustness Principles for Public Key Protocols. In: Coppersmith, D. (ed.) CRYPTO 1995. LNCS, vol. 963, pp. 236-247. Springer, Heidelberg (1995)

2. Anderson, R.J.: Security Engineering: A Guide to Building Dependable Distributed Systems, 2nd edn. Wiley (2008)

3. Boyen, X., Dodis, Y., Katz, J., Ostrovsky, R., Smith, A.: Secure Remote Authentication Using Biometric Data. In: Cramer, R. (ed.) EUROCRYPT 2005. LNCS, vol. 3494, pp. 147-163. Springer, Heidelberg (2005); Lee, J.K., Ryu, S.R.: Fingerprint-based Remote User Authentication Scheme Using Smart Cards. Electronics Leters 38(12), 554-555 (2005)

4. Boyd, C., Mathuria, A.: Protocols for Authentication and Key Establishment. Springer (2003)

5. Boyen, X.: Reusable Cryptographic Fuzzy Extractors. In: ACM CCS 2004, pp. 82-91 (2004)

6. Dodis, Y., Reyzin, L., Smith, A.: Fuzzy Extractors: How to Generate Strong Keys from Biometrics and Other Noisy Data. In: Cachin, C., Camenisch, J.L. (eds.) EUROCRYPT 2004. LNCS, vol. 3027, pp. 523-540. Springer, Heidelberg (2004)

7. Hao, F.: On Robust Key Agreement Based on Public Key Authentication. In: Sion, R. (ed.) FC 2010. LNCS, vol. 6052, pp. 383-390. Springer, Heidelberg (2010)

8. Hao, F., Anderson, R., Daugman, J.: Combining crypto with biometrics effectively. IEEE Transactions on Computers 55(9), 1081-1088 (2006)

9. Krawczyk, H.: HMQV: A High-Performance Secure Diffie-Hellman Protocol. In: Shoup, V. (ed.) CRYPTO 2005. LNCS, vol. 3621, pp. 546-566. Springer, Heidelberg (2005) 
10. Hwang, M., Chong, S., Chen, T.: DoS-Resistant ID-Based Password Authentication Scheme Using Smart Cards. Computer Journal of Systems and Software 7(50), 147-150 (2009)

11. Lee, Y., Kim, S., Won, D.: Enhancement of Two-Factor Authenticated Key Exchange Protocols in Public Wireless LANs. Computers and Electrical Engineering 36(1), 213-223 (2010)

12. Lian, I.E., Lee, C.C., Hwang, M.S.: A Password Authentication Scheme Over Insecure Networks. Journal of Computer System Sciences 72, 727-740 (2006)

13. Li, C.T., Hwang, M.S.: An Efficient Biometrics-Based Remote User Authentication Scheme Using Smart Cards. Journal of Network and Computer Applications 33(1), $1-5(2010)$

14. Li, X., Niu, J.W., Ma, J., Wang, W.D.: Cryptanalysis and Improvement of a Biometrics-Based Remote User Authentication Scheme Using Smart Cards. Journal of Network and Computer Applications 34(1), 73-79 (2011)

15. Liu, Y., Wei, F., Ma, C.: Multi-Factor Authenticated Key Exchange Protocol in the Three-Party Setting. In: Lai, X., Yung, M., Lin, D. (eds.) Inscrypt 2010. LNCS, vol. 6584, pp. 255-267. Springer, Heidelberg (2011)

16. Menezes, A.J., van Oorschot, P.C., Vanstone, S.A.: Handbook of Applied Cryptography. CRC Press (1996)

17. Menezes, A., Ustaoglu, B.: On the Importance of Public-Key Validation in the MQV and HMQV Key Agreement Protocols. In: Barua, R., Lange, T. (eds.) INDOCRYPT 2006. LNCS, vol. 4329, pp. 133-147. Springer, Heidelberg (2006)

18. Pointcheval, D., Zimmer, S.: Multi-factor Authenticated Key Exchange. In: Bellovin, S.M., Gennaro, R., Keromytis, A.D., Yung, M. (eds.) ACNS 2008. LNCS, vol. 5037, pp. 277-295. Springer, Heidelberg (2008)

19. Song, R.: Advanced Smart Card Based Password Authentication Protocol. Computer Standards \& Interfaces 32, 321-325 (2010)

20. Tapiador, J.E., Hernandez-Castro, J.C., Peris-Lopez, P., Clark, J.A.: Cryptanalysis of Song's Advanced Smart Card Based Password Authentication Protocol (2011), Technical report available at http://arxiv.org/pdf/1111.2744

21. Wu, S., Zhu, Y.: Improved Two-Factor Authenticated Key Exchange Protocol. The International Arab Journal of Information Technology 8(4), 430-439 (2011)

22. Xiang, T., Wong, K., Liao, X.: Cryptanalysis of A Password Authentication Scheme Over Insecure Networks. Journal of Computer System Sciences 74, 657-661 (2008)

23. Xu, J., Zhu, W.T., Feng, D.G.: An Improved Smart Card Based Password Authentication Scheme with Provable Security. Computer Standards \& Interfaces 31, $723-728$ (2009) 